\title{
Relationship between Salt Preference and Gastric Cancer Screening: An Analysis of a Nationwide Survey in Korea
}

Ji-Yeon Shin, $M D, P h D^{1}$
Jeongseon Kim, $\mathrm{PhD}^{2}$
Kui Son Choi, $\mathrm{PhD}^{2,3}$
Mina Suh, MD, $\mathrm{PhD}^{3}$
Boyoung Park, MD, $\mathrm{PhD}^{2,3}$
Jae Kwan Jun, $\mathrm{MD}, \mathrm{PhD}^{2,3}$

${ }^{1}$ Department of Preventive Medicine, Eulji University School of Medicine, Daejeon, ${ }^{2}$ Graduate School of Cancer Science and Policy, National Cancer Center, Goyang, ${ }^{3}$ National Cancer Control Institute, National Cancer Center, Goyang, Korea

\section{Correspondence: Jae Kwan Jun, MD, PhD}

National Cancer Control Institute,

National Cancer Center, 323 Ilsan-ro,

Ilsandong-gu, Goyang 10408, Korea

Tel: 82-31-920-2184

Fax: 82-31-920-2949

E-mail: jkjun@ncc.re.kr

Received September 1, 2015

Accepted November 16, 2015

Published Online December 11, 2015

\begin{abstract}
Purpose
Epidemiological studies have demonstrated an association between excessive salt intake and gastric cancer risk, and this potential risk increases the need for adequate gastric cancer screening in individuals with high salt intake. However, the association between salt intake and gastric cancer screening in the general population has rarely been investigated. We explored the association between salt preference and participation in gastric cancer screening among a nationally representative Korean population.
\end{abstract}

\section{Materials and Methods}

The study population was derived from the Korean National Cancer Screening Survey (KNCSS) 2006-2007, an annual nationwide interview survey investigating cancer screening rates. Of 4,055 individuals who participated in the KNCSS 2006-2007, 3,336 individuals aged over 40 years were included in our analysis. The odds ratio (OR) and $95 \%$ confidence interval $(\mathrm{Cl})$ were estimated using polytomous logistic regression.

\section{Results}

Individuals with higher salt preference were less likely to participate in regular gastric cancer screening. After adjusting for age, sex, monthly household income, education, family history of cancer, and self-rated health status, ORs for undergoing regular gastric cancer screening were $1.00,0.82(95 \% \mathrm{Cl}, 0.61$ to 1.12$), 0.74(95 \% \mathrm{Cl}, 0.54$ to 1.00$), 0.77(95 \% \mathrm{Cl}, 0.56$ to 1.05), and $0.38(95 \% \mathrm{Cl}, 0.16$ to 0.92$)$ according to the level of salt preference ( $\mathrm{p}$ for trend $=0.048$ ).

\section{Conclusion}

Individuals with higher salt preference showed suboptimal gastric cancer screening adherence compared to those with a lower salt preference. These findings highlight the need for better delivery of educational messages to change risk perceptions regarding gastric cancer screening practice.

\section{Introduction}

Gastric cancer is the third leading cause of cancer-related mortality worldwide, with 952,000 new cases and 723,000 deaths per year [1]. In Korea, despite decreasing incidence and mortality of gastric cancer, it remains the most common cancer in men, with an age-standardized incidence rate of
Key words

Stomach neoplasms, Early detection of cancer, Dietary sodium 
advanced gastric cancer [4]. Japan and Korea, where gastric cancer is highly prevalent, have national guidelines or recommendations for gastric cancer screening and have been providing mass screening to average-risk populations $[3,5]$. In Korea, the National Cancer Screening Program (NCSP) recommends biennial upper gastrointestinal series (UGIS) or upper endoscopy for men and women aged $>40$ years [5].

Understanding the risk factors associated with gastric carcinogenesis is important for identification of high-risk groups that may need screening [3]. Excessive salt intake is known to be associated with gastric cancer risk [3]. Evidence from prospective studies suggests that high salt intake or salt preference increases the risk of gastric cancer [6,7]. Excessive salt is considered to have a dual effect at the initial stages of the chain of causation, inducing both gastritis and atrophy. It may also affect the later steps of carcinogenesis, progressing through the stages of intestinal metaplasia and dysplasia and eventually resulting in carcinoma [8]. This potential risk increases the need for adequate gastric cancer screening in individuals with high salt preference. Nevertheless, to the best of our knowledge, no studies on the gastric cancer screening status of this population as a high-risk group have been conducted. Thus, we investigated the association between participation in gastric cancer screening and salt preference, adding to known relevant factors including sociodemographic and cognitive factors among a nationally representative Korean population.

\section{Materials and Methods}

Data were obtained from the Korean National Cancer Screening Survey (KNCSS) 2006-2007. KNCSS is a nationwide representative interview survey conducted annually by the Korean National Cancer Center since 2004 to examine the participation rates of Koreans in cancer screening for five common cancers: gastric, liver, colorectal, breast, and cervical. Men and women are selected based on the Resident Registration Population data using a stratified, multistage, random sampling procedure according to geographic area, age, and sex. The Resident Registration Population data are published annually by the Korea National Statistical Office after collection of data from residents of the registration population every December 31. Five to eight households in an urban area and 10 to 12 households in a rural area were randomly chosen. Investigators from a professional research agency conducted face-to-face interviews in the participants' homes. Participants were recruited through door-to-door contact. At least three attempts were made to contact a resident at each dwelling. Eligible participants were asked about their experiences of screening for five common cancers and provided information on health behaviors, health status, family history of cancer, and sociodemographic factors. Details of the survey have been described elsewhere [5]. This study was approved by the Institutional Review Board of the National Cancer Center, Korea.

Interviews were completed by 2,033 participants in 2006 and 2,022 participants in 2007 aged 30 years or older (response rate, $43.4 \%$ and $33.3 \%$, respectively). Our analyses were limited to cancer-free participants over the age of 40 years. A total of 3,336 participants were selected as final study subjects. All subjects provided written informed consent for participation in the study.

Participants were asked the following questions: "Have you ever been screened for gastric cancer?", "When was the last time you had a gastric cancer examination?", and "Which tests did you undergo (UGIS, upper endoscopy, or both)?" "Lifetime gastric cancer screening" was defined as having undergone gastric cancer screening at least once in one's lifetime, irrespective of the screening interval or method. "Compliance with the recommendations" was defined as having undergone gastric cancer screening within the previous 2 years according to NCSP recommendations, and "noncompliance with the recommendation" was defined as having undergone gastric cancer screening more than 2 years ago.

Participants were also asked about their salt preference; we posed the question: "Do you like salty food?" Participants chose from one of five graded responses: hate, not like, moderate, like, or love. Based on this response, salt preference was classified according to five categories: very low, low, moderate, high, and very high. Age (40-49, 50-59, or $\geq 60$ years), sex, monthly household income $(<1,500,1,500-2,499$, $2,500-3,499$, or $\geq 3,500$ USD), education ( $\leq 11,12-15$, or $\geq 16$ years), family history of cancer (no or yes), and self-rated health status (good, fair, or bad) were considered as potential confounders.

Basic characteristics of the study population by lifetime gastric cancer screening are presented as percentages. Univariate and multivariate logistic regression were performed to examine the association between salt preference and lifetime gastric cancer screening. Multivariable polytomous logistic regression models were used for calculation of adjusted odds ratios (aORs) and 95\% confidence intervals (CIs) for compliance and noncompliance with gastric cancer screening recommendations compared with never-screening according to salt preference level. The association between salt preference and gastric cancer screening practice according to the screening modality (UGIS vs. upper endoscopy or upper endoscopy+UGIS) compared with never-screening in the study population was also assessed using multivariable polytomous logistic regression. Statistical analyses were per- 
formed using SAS ver. 9.2 (SAS Institute Inc., Cary, NC). Sample weights were incorporated into all SAS survey procedures to ensure the correct estimation of sampling error. All reported p-values are two-sided, and those that were $<0.05$ were considered statistically significant.

\section{Results}

Of the 3,336 survey respondents, 1,723 were female and 1,613 were male. A total of 1,521 subjects reported they had never undergone gastric cancer screening, and 1,815 subjects reported they had undergone screening for gastric cancer at least once. Baseline characteristics, odds ratios (ORs), and 95\% CIs for lifetime gastric cancer screening among the study population are shown in Table 1. Compared with the neverscreened group, subjects in the lifetime gastric cancer screening group were more likely to be female, older, live in a rural area, less educated, and have a worse self-rated health status. According to bivariate analysis, subjects with a higher salt preference were less likely to participate in gastric cancer screening, although this difference did not reach statistical significance (crude OR, 0.52 for "very high"; $95 \%$ CI, 0.25 to 1.07) (Table 1). After adjusting for covariates, the odds of undergoing gastric cancer screenings in their lifetime remained significantly reduced for subjects with a high salt preference. The aORs across the levels of salt preference were 1.00, 0.79 (95\% CI, 0.59 to 1.06), 0.71 (95\% CI, 0.53 to 0.95), 0.75 (95\% CI, 0.56 to 1.01), and 0.44 (95\% CI, 0.20 to 0.97$)$ (p for trend $=0.052$ ) (Table 1).

Results of polytomous logistic regression analyses for associations of salt preference with compliance and noncompliance with NCSP recommendations compared with neverscreening are shown in Table 2. Subjects with a higher salt preference were less likely to participate in regular gastric cancer screening according to the recommendations. The aORs were 1.00, 0.82 (95\% CI, 0.61 to 1.12), 0.74 (95\% CI, 0.54 to 1.00$), 0.77$ ( $95 \% \mathrm{CI}, 0.56$ to 1.05 ), and 0.38 (95\% CI, 0.16 to 0.92 ) according to the level of salt preference ( $\mathrm{p}$ for trend= 0.048). For noncompliance with recommended gastric cancer screening, lower odds were observed for a moderate salt preference than for a very low salt preference (aOR, 0.60; 95\% CI, 0.37 to 0.98 ), but the trend did not reach statistical significance.

Associations between salt preference and gastric cancer screening according to screening modalities compared with never-screening are shown in Table 3. The adjusted odds of undergoing gastric cancer screening with UGIS were 0.56 (95\% CI, 0.36 to 0.86 ) for low, 0.53 (95\% CI, 0.34 to 0.82 ) for moderate, 0.58 (95\% CI, 0.37 to 0.90$)$ for high, and $0.32(95 \%$
CI, 0.09 to 1.21 ) for very high salt preference compared with very low salt preference ( $\mathrm{p}$ for trend $=0.121$ ). With respect to gastric cancer screening with upper endoscopy, we also observed lower odds of having a higher salt preference than having a very low salt preference (aORs across the levels were $1.00,0.87,0.77,0.81$, and 0.49 , respectively), although the trend was not statistically significant ( $\mathrm{p}$ for trend $=0.125$ ).

\section{Discussion}

In this population-based study conducted in Korea, a high salt preference was associated with a maximum $60 \%$ decreased likelihood of regular participation in gastric cancer screening according to NCSP recommendations. To the best of our knowledge, this is the first study to evaluate an association between salt preference and gastric cancer screening using a nationally representative sample.

According to the report of a joint World Health Organization (WHO)/Food and Agriculture Organization of the United Nations (FAO) Expert Consultation, salt-preserved foods and salt probably increase the risk of stomach cancer [9]. Epidemiological and experimental evidence consistently supports the association between salt intake and gastric cancer risk. Several population-based prospective studies have reported significant association of high salt intake with increased risk of gastric cancer [6,7]. Experimental studies have shown that a high intragastric salt concentration alters the viscosity of the protective mucosal barrier [10], leading to mucosal damage and inflammation [11]. Persistent inflammatory changes can promote temporary cell proliferation and increase the rate of endogenous mutations [11]. An excessively salty diet induced atrophy in experimental animals and was associated with atrophic changes in the human gastric mucosa [8], thereby possibly increasing the risk of gastric cancer.

In addition, there is evidence of synergistic interactions between salt intake and Helicobacter pylori infection with respect to the development of gastric cancer. In an experimental study of gerbils, a high-salt diet enhanced the effects of $H$. pylori infection on gastric carcinogenesis, and these two factors acted synergistically to promote the development of gastric cancer [12]. A prospective study showed that the effect of high salt intake on gastric carcinogenesis was strong in subjects who had both $H$. pylori infection and atrophic gastritis [7]. Excessive salt intake has been suggested as facilitating H. pylori colonization [7].

Considering all the evidence suggesting that excessive salt intake increases the risk of gastric cancer, individuals with high salt intake can benefit from regular cancer screening. 
Table 1. Study population characteristics, odds ratios, and 95\% CI for lifetime gastric cancer screening among pooled 20062007 Korea National Cancer Screening Survey $(n=3,336)$

\begin{tabular}{|c|c|c|c|c|c|c|}
\hline \multirow{3}{*}{ Variable } & \multicolumn{2}{|c|}{ Lifetime gastric cancer screening (weighted \%) } & \multirow{3}{*}{ cOR $(95 \% \mathrm{CI})$} & \multirow{3}{*}{$p$ for trend } & \multirow{3}{*}{$\mathrm{aOR}^{\mathrm{a})}(95 \% \mathrm{CI})$} & \multirow{3}{*}{$p$ for trend } \\
\hline & No & Yes & & & & \\
\hline & \multicolumn{2}{|c|}{ (unweighted n=1,521) (unweighted n=1,815) } & & & & \\
\hline \multicolumn{7}{|l|}{ Sex } \\
\hline Male & 52.4 & 44.9 & 1.00 (reference) & - & 1.00 (reference) & - \\
\hline Female & 47.6 & 55.1 & $1.36(1.18-1.55)$ & & $1.29(1.12-1.49)$ & \\
\hline \multicolumn{7}{|l|}{ Age group (yr) } \\
\hline $40-49$ & 49.8 & 35.7 & 1.00 (reference) & $<0.001$ & 1.00 (reference) & $<0.001$ \\
\hline $50-59$ & 23.9 & 28.5 & $1.66(1.40-1.97)$ & & $1.71(1.42-2.05)$ & \\
\hline$\geq 60$ & 26.3 & 35.8 & $1.90(1.62-2.24)$ & & $2.02(1.64-2.49)$ & \\
\hline \multicolumn{7}{|l|}{ Residential area } \\
\hline Metropolitan & 49.1 & 45.1 & 1.00 (reference) & 0.003 & 1.00 (reference) & 0.001 \\
\hline Urban & 39.7 & 40.6 & $1.12(0.96-1.29)$ & & $1.16(1.00-1.36)$ & \\
\hline Rural & 11.2 & 14.3 & $1.39(1.12-1.73)$ & & $1.44(1.14-1.81)$ & \\
\hline \multicolumn{7}{|c|}{ Monthly household income } \\
\hline$\leq 1,499$ USD & 26.7 & 28.5 & 1.00 (reference) & 0.878 & 1.00 (reference) & $<0.001$ \\
\hline 1,500-2,499 USD & 30.2 & 28.1 & $0.87(0.73-1.05)$ & & $1.25(1.02-1.53)$ & \\
\hline 2,500-3,499 USD & 24.9 & 24.3 & $0.92(0.76-1.11)$ & & $1.52(1.21-1.90)$ & \\
\hline$\geq 3,500$ USD & 18.3 & 19.2 & $0.98(0.80-1.21)$ & & $1.54(1.20-1.96)$ & \\
\hline \multicolumn{7}{|l|}{ Education (yr) } \\
\hline$\leq 11$ & 36.2 & 45.1 & 1.00 (reference) & $<0.001$ & 1.00 (reference) & 0.693 \\
\hline $12-15$ & 46.5 & 38.6 & $0.67(0.57-0.78)$ & & $0.90(0.74-1.09)$ & \\
\hline$\geq 16$ & 17.3 & 16.3 & $0.76(0.62-0.92)$ & & $1.07(0.84-1.37)$ & \\
\hline \multicolumn{7}{|c|}{ Family history of cancer } \\
\hline No & 91.7 & 87.7 & 1.00 (reference) & - & 1.00 (reference) & - \\
\hline Yes & 8.3 & 10.3 & $1.27(1.01-1.61)$ & & $1.28(1.00-1.63)$ & \\
\hline \multicolumn{7}{|l|}{ Self-rated health status } \\
\hline Good & 66.5 & 55.2 & 1.00 (reference) & $<0.001$ & 1.00 (reference) & $<0.001$ \\
\hline Fair & 25.3 & 31.2 & $1.48(1.27-1.73)$ & & $1.49(1.26-1.74)$ & \\
\hline Poor & 8.2 & 13.6 & $2.00(1.59-2.53)$ & & $1.81(1.42-2.31)$ & \\
\hline \multicolumn{7}{|l|}{ Salt preference } \\
\hline Level 1 (very low) & 6.5 & 8.3 & 1.00 (reference) & 0.283 & 1.00 (reference) & 0.052 \\
\hline Level 2 (low) & 35.9 & 36.1 & $0.78(0.59-1.03)$ & & $0.79(0.59-1.06)$ & \\
\hline Level 3 (moderate) & 29.2 & 26.4 & $0.71(0.53-0.94)$ & & $0.71(0.53-0.95)$ & \\
\hline Level 4 (high) & 27.2 & 28.3 & $0.81(0.61-1.08)$ & & $0.75(0.56-1.01)$ & \\
\hline Level 5 (very high) & 1.3 & 0.8 & $0.52(0.25-1.07)$ & & $0.44(0.20-0.97)$ & \\
\hline
\end{tabular}

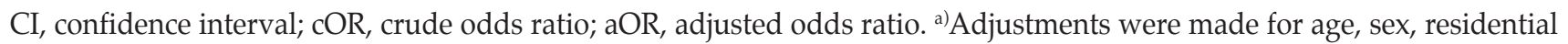
area, monthly household income, education, family history of cancer, and self-rated health status.

However, our results showed that individuals with a higher salt preference, who may have a higher risk of developing gastric cancer, were less likely to participate in gastric cancer screening regardless of screening regularity or modality. In addition, the odds of undergoing screening by upper endoscopy in the higher salt preference group were suboptimal compared with the low salt preference group. This was despite the fact that upper endoscopy can be a very helpful screening modality for this group because it can identify minute mucosal lesions and nonulcerative lesions as well as superficial flat lesions that can be missed during conventional barium examination [3].

The act of participating in cancer screening is the culmination of a complex chain of events involving many factors, including some related to the person being screened, that person's family and social surroundings, the health care system, and society at large [13]. However, even if equitable access can be ensured, individual-specific factors remain 
Table 2. aORs and 95\% CI for compliance and noncompliance with gastric cancer screening recommendations compared with never-screening by polytomous logistic regression

\begin{tabular}{|c|c|c|c|c|}
\hline \multirow[t]{2}{*}{ Variable } & \multicolumn{2}{|c|}{$\begin{array}{l}\text { Noncompliance with the recommendation } \\
(>2 \text { years) (unweighted } n=332 \text { ) }\end{array}$} & \multicolumn{2}{|c|}{$\begin{array}{l}\text { Compliance with the recommendation } \\
\text { (within } 2 \text { years) (unweighted } n=1,483 \text { ) }\end{array}$} \\
\hline & $\mathrm{aOR}^{\mathrm{a})}(95 \% \mathrm{CI})$ & $\mathrm{p}$ for trend & $\mathrm{aOR}^{\mathrm{a})}(95 \% \mathrm{CI})$ & $\mathrm{p}$ for trend \\
\hline \multicolumn{5}{|l|}{ Sex } \\
\hline Male & 1.00 (reference) & - & 1.00 (reference) & - \\
\hline Female & $1.31(1.02-1.69)$ & & $1.29(1.11-1.50)$ & \\
\hline \multicolumn{5}{|l|}{ Age group (yr) } \\
\hline $40-49$ & 1.00 (reference) & $<0.001$ & 1.00 (reference) & $<0.001$ \\
\hline $50-59$ & $1.85(1.36-2.53)$ & & $1.68(1.38-2.03)$ & \\
\hline$\geq 60$ & $1.87(1.33-2.63)$ & & $2.06(1.65-2.56)$ & \\
\hline \multicolumn{5}{|l|}{ Residential area } \\
\hline Metropolitan & 1.00 (reference) & 0.071 & 1.00 (reference) & $<0.001$ \\
\hline Urban & $1.31(1.01-1.70)$ & & $1.13(0.97-1.33)$ & \\
\hline Rural & $1.54(1.05-2.24)$ & & $1.42(1.11-1.80)$ & \\
\hline \multicolumn{5}{|c|}{ Monthly household income } \\
\hline$\leq 1,499$ USD & 1.00 (reference) & 0.199 & 1.00 (reference) & 0.001 \\
\hline 1,500-2,499 USD & $1.28(0.91-1.79)$ & & $1.25(1.01-1.54)$ & \\
\hline 2,500-3,499 USD & $1.43(0.98-2.09)$ & & $1.54(1.22-1.95)$ & \\
\hline$\geq 3,500$ USD & $1.45(0.97-2.17)$ & & $1.56(1.20-2.01)$ & \\
\hline \multicolumn{5}{|l|}{ Education (yr) } \\
\hline$\leq 11$ & 1.00 (reference) & 0.460 & 1.00 (reference) & 0.601 \\
\hline $12-15$ & $0.92(0.67-1.25)$ & & $0.89(0.73-1.09)$ & \\
\hline$\geq 16$ & $0.93(0.62-1.40)$ & & $1.10(0.85-1.43)$ & \\
\hline \multicolumn{5}{|c|}{ Family history of cancer } \\
\hline No & 1.00 (reference) & - & 1.00 (reference) & - \\
\hline Yes & $1.15(0.75-1.76)$ & & $1.31(1.02-1.69)$ & \\
\hline \multicolumn{5}{|l|}{ Self-rated health status } \\
\hline Good & 1.00 (reference) & $<0.001$ & 1.00 (reference) & $<0.001$ \\
\hline Fair & $1.49(1.13-1.96)$ & & $1.49(1.26-1.76)$ & \\
\hline Poor & $2.50(1.74-3.59)$ & & $1.66(1.29-2.15)$ & \\
\hline \multicolumn{5}{|l|}{ Salt preference } \\
\hline Level 1 (very low) & 1.00 (reference) & 0.681 & 1.00 (reference) & 0.048 \\
\hline Level 2 (low) & $0.66(0.42-1.05)$ & & $0.82(0.61-1.12)$ & \\
\hline Level 3 (moderate) & $0.60(0.37-0.98)$ & & $0.74(0.54-1.00)$ & \\
\hline Level 4 (high) & $0.68(0.42-1.10)$ & & $0.77(0.56-1.05)$ & \\
\hline Level 5 (very high) & $0.68(0.23-1.99)$ & & $0.38(0.16-0.92)$ & \\
\hline
\end{tabular}

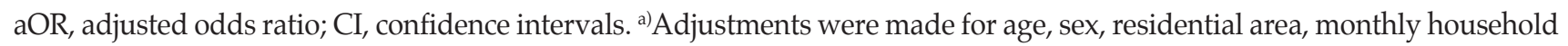
income, education, family history of cancer, and self-rated health status.

important [13]. That is, even if there were no barriers to cancer screening, individuals still need to decide for themselves whether or not to undergo screening. Risk perception is one of the most important factors in health-related decision making. In fact, cancer risk perceptions have been suggested to be a key predictor of risk-reduction practices, health behaviors, and processing of cancer information [14]. Significant evidence has indicated that risk perceptions are related to health behavior and medical decision making. Kinney et al.
[15] reported that participants who believed they had an average or reduced risk of colorectal cancer were less likely to have undergone recent endoscopic surveillance of the colorectum than those who believed they were at greater risk. McCaul et al. [16] reported that women with higher risk perceptions for breast cancer were more likely to undergo mammography screening. In our study, the high salt preference group may not have been aware of excessive salt intake as a risk for development of gastric cancer. If individuals recog- 
Table 3. aORs and 95\% CI for undergoing gastric cancer screening with upper gastrointestinal series and with upper endoscopy compared with never-screening by polytomous logistic regression

\begin{tabular}{|c|c|c|c|c|}
\hline \multirow[t]{2}{*}{ Variable } & \multicolumn{2}{|c|}{$\begin{array}{l}\text { Upper gastrointestinal series } \\
\quad \text { (unweighted } n=355 \text { ) }\end{array}$} & \multicolumn{2}{|c|}{$\begin{array}{l}\text { Upper endoscopy } \\
\text { (unweighted } n=1,460 \text { ) }\end{array}$} \\
\hline & $\mathrm{aOR}^{\mathrm{a})}(95 \% \mathrm{CI})$ & $\mathrm{p}$ for trend & $\left.\mathrm{aOR}^{\mathrm{a}}\right)(95 \% \mathrm{CI})$ & $p$ for trend \\
\hline \multicolumn{5}{|l|}{ Sex } \\
\hline Male & 1.00 (reference) & - & 1.00 (reference) & - \\
\hline Female & $1.30(1.02-1.66)$ & & $1.29(1.11-1.50)$ & \\
\hline \multicolumn{5}{|l|}{ Age group (yr) } \\
\hline $40-49$ & 1.00 (reference) & $<0.001$ & 1.00 (reference) & $<0.001$ \\
\hline $50-59$ & $1.49(1.08-2.04)$ & & $1.76(1.45-2.13)$ & \\
\hline$\geq 60$ & $1.86(1.33-2.61)$ & & $2.06(1.65-2.56)$ & \\
\hline \multicolumn{5}{|l|}{ Residential area } \\
\hline Metropolitan & 1.00 (reference) & $<0.001$ & 1.00 (reference) & 0.008 \\
\hline Urban & $1.20(0.92-1.56)$ & & $1.16(0.99-1.36)$ & \\
\hline Rural & $2.28(1.63-3.18)$ & & $1.24(0.96-1.58)$ & \\
\hline \multicolumn{5}{|c|}{ Monthly household income } \\
\hline$\leq 1,499$ USD & 1.00 (reference) & 0.867 & 1.00 (reference) & $<0.001$ \\
\hline 1,500-2,499 USD & $1.16(0.84-1.60)$ & & $1.28(1.04-1.59)$ & \\
\hline 2,500-3,499 USD & $1.00(0.68-1.47)$ & & $1.68(1.32-2.12)$ & \\
\hline$\geq 3,500$ USD & $1.06(0.71-1.59)$ & & $1.68(1.30-2.18)$ & \\
\hline \multicolumn{5}{|l|}{ Education (yr) } \\
\hline$\leq 11$ & 1.00 (reference) & 0.742 & 1.00 (reference) & 0.879 \\
\hline $12-15$ & $0.74(0.54-1.01)$ & & $0.94(0.77-1.15)$ & \\
\hline$\geq 16$ & $1.05(0.71-1.56)$ & & $1.08(0.83-1.40)$ & \\
\hline \multicolumn{5}{|c|}{ Family history of cancer } \\
\hline No & 1.00 (reference) & - & 1.00 (reference) & - \\
\hline Yes & $1.22(0.81-1.85)$ & & $1.29(1.00-1.67)$ & \\
\hline \multicolumn{5}{|l|}{ Self-rated health status } \\
\hline Good & 1.00 (reference) & 0.574 & 1.00 (reference) & $<0.001$ \\
\hline Fair & $1.27(0.97-1.67)$ & & $1.54(1.30-1.82)$ & \\
\hline Poor & $1.32(0.89-1.96)$ & & $1.95(1.52-2.52)$ & \\
\hline \multicolumn{5}{|l|}{ Salt preference } \\
\hline Level 1 (very low) & 1.00 (reference) & 0.121 & 1.00 (reference) & 0.125 \\
\hline Level 2 (low) & $0.56(0.36-0.86)$ & & $0.87(0.64-1.19)$ & \\
\hline Level 3 (moderate) & $0.53(0.34-0.82)$ & & $0.77(0.56-1.06)$ & \\
\hline Level 4 (high) & $0.58(0.37-0.90)$ & & $0.81(0.59-1.12)$ & \\
\hline Level 5 (very high) & $0.32(0.09-1.21)$ & & $0.49(0.21-1.12)$ & \\
\hline
\end{tabular}

aOR, adjusted odds ratio; $\mathrm{CI}$, confidence interval. a)Adjustments were made for age, sex, residential area, monthly household income, education, family history of cancer, and self-rated health status.

nize that their dietary habits influence gastric cancer risk, their screening behaviors are more likely to change.

In addition, the higher salt preference group may not have had sufficient knowledge on gastric cancer, including its risk factors and the importance of screening practices. To some extent, risk awareness is associated with knowledge of cancer risk. In a study of colorectal cancer screening, lack of knowledge and awareness was a reported reason for not having undergone screening [17]. Groups with a better understand- ing of the risk factors for a disease and the benefits to be gained by implementing recommended health behaviors tend to choose healthier behaviors.

Our findings may also be explained in part by an optimistic bias; people often judge themselves to be at lower risk for various negative life events than do their peers, despite their unhealthy behaviors [18]. This can prevent people from participating in appropriate health behaviors that may reduce their risk of becoming ill. For example, smokers who 
underestimate their lung cancer risk are also more likely to endorse beliefs supportive of smoking (e.g., there is no risk of lung cancer if one only smokes for a few years) and less likely to plan on quitting smoking [19]. People who prefer a salty taste may underestimate their gastric cancer risk, have less knowledge about the causes of gastric cancer, worry less about developing gastric cancer, and be less likely to participate in screening behavior [14].

Collectively, our study findings support the need for delivery of educational messages to change risk perceptions for people with risk factors. This may be best accomplished by both health care system-based initiatives and media campaigns. In addition, individuals should be provided with information regarding the facts that those with higher salt preference can benefit from upper endoscopy screening, that excessive salt intake is related to $H$. pylori infection, and people who have these two factors concurrently should be more aware of undergoing regular gastric cancer screening. Personalized cancer risk assessment with consideration of one's various risk factors and recommendations for selecting optimal screening modalities or intervals may be helpful in the clinical setting. Fortunately, salt preference can be easily assessed in the clinical setting and is useful for dietary intervention [20].

Previous studies of the Korean population have suggested that participation in the gastric cancer screening program is affected by various factors, including socio-demographic, health behavior, and community level factors. Younger age [21], lower education level [21-23], lower income level $[22,23]$, living without a spouse [21,22], excessive alcohol consumption [21,23], and currently smoking $[21,22]$ were associated with less participation in gastric cancer screening. Urbanization as a community-level factor also showed a negative association with undergoing gastric cancer screening [22].

It is important to note that other health behavioral factors, such as smoking and alcohol consumption, were not included as covariates. Close association of healthy dietary habits with other good health behaviors has been reported [24]. In this study, we found that those who frequently drank or currently smoked cigarettes were more likely to belong to the high salt preference group ( $\mathrm{p}=0.042$ and $\mathrm{p}=0.002$, respectively, by Mantel-Haenszel chi-squared test; data not shown). Because collinearity between salt preference and other health behaviors could interrupt or dilute the association we sought to identify, we did not include other health behavioral factors in the multivariable model.

This study had several limitations. First, we could not measure salt intake; instead, we used salt preference, which was self-reported in a questionnaire. Therefore, we could not present exact sodium quantities in $\mathrm{mg} /$ day. However, previous studies have indicated that a high salt preference may result in high long-term sodium intake because salt preference is considered to be formed by food selection $[6,20]$. In addition, because salt preference is reflective of an individual's attitude and response to salty food, it may be useful for the qualitative evaluation of salt intake [20]. In fact, a recent study showed considerable correlation between salt taste preference and 24-hour urinary sodium level [25]. Second, the KNCSS data were self-reported. Therefore, some inaccuracy due to inaccurate reporting by respondents may have occurred. In particular, salt preference was defined via a single item in the self-reported questionnaire, and could have resulted in some misclassification. However, misclassification of salt preference would likely be nondifferential and would have led to a bias toward the null. Third, we were unable to explore other factors that might have affected gastric cancer screening, such as $H$. pylori infection status as a risk factor for gastric cancer and physician factors such as doctors' recommendations. Fourth, because of the cross-sectional design we cannot rule out the possibility of reverse causation.

\section{Conclusion}

In this nationwide survey conducted in Korea, individuals with higher salt preference showed suboptimal gastric cancer screening adherence compared with individuals with a lower salt preference. These findings highlight the need for better delivery of educational messages to change risk perceptions regarding gastric cancer screening practice. Individually tailored risk assessment, appropriate risk education, and proper recommendations for screening options are needed for informed decision making.

\section{Conflicts of Interest}

Conflict of interest relevant to this article was not reported.

\section{Acknowledgments}

This work was supported by a grant from the National R\&D Program for Cancer Control, No. 1310232. 


\section{References}

1. International Agency for Research on Cancer. GLOBOCAN 2012: estimated cancer incidence, mortality and prevalence worldwide in 2012 [Internet]. Lyon: IARC; 2015 [cited 2015 Jun 7]. Available from: http://globocan.iarc.fr/Pages/fact_sheets_ cancer.aspx.

2. Jung KW, Won YJ, Kong HJ, Oh CM, Cho H, Lee DH, et al. Cancer statistics in Korea: incidence, mortality, survival, and prevalence in 2012. Cancer Res Treat. 2015;47:127-41.

3. Leung WK, Wu MS, Kakugawa Y, Kim JJ, Yeoh KG, Goh KL, et al. Screening for gastric cancer in Asia: current evidence and practice. Lancet Oncol. 2008;9:279-87.

4. Lee KJ, Inoue M, Otani T, Iwasaki M, Sasazuki S, Tsugane S, et al. Gastric cancer screening and subsequent risk of gastric cancer: a large-scale population-based cohort study, with a 13year follow-up in Japan. Int J Cancer. 2006;118:2315-21.

5. Suh M, Choi KS, Lee YY, Jun JK. Trends in cancer screening rates among Korean men and women: results from the Korean national cancer screening survey, 2004-2012. Cancer Res Treat. 2013;45:86-94.

6. Kim J, Park S, Nam BH. Gastric cancer and salt preference: a population-based cohort study in Korea. Am J Clin Nutr. 2010;91:1289-93.

7. Shikata K, Kiyohara Y, Kubo M, Yonemoto K, Ninomiya T, Shirota T, et al. A prospective study of dietary salt intake and gastric cancer incidence in a defined Japanese population: the Hisayama study. Int J Cancer. 2006;119:196-201.

8. Correa P. Human gastric carcinogenesis: a multistep and multifactorial process: first American Cancer Society Award Lecture on Cancer Epidemiology and Prevention. Cancer Res. 1992;52:6735-40.

9. World Health Organization. Diet, nutrition and the prevention of chronic diseases. WHO technical report series 916. Geneva: World Health Organization; 2003.

10. Tatematsu M, Takahashi M, Fukushima S, Hananouchi M, Shirai $\mathrm{T}$. Effects in rats of sodium chloride on experimental gastric cancers induced by N-methyl-N-nitro-N-nitrosoguanidine or 4-nitroquinoline-1-oxide. J Natl Cancer Inst. 1975;55:101-6.

11. Furihata C, Ohta H, Katsuyama T. Cause and effect between concentration-dependent tissue damage and temporary cell proliferation in rat stomach mucosa by $\mathrm{NaCl}$, a stomach tumor promoter. Carcinogenesis. 1996;17:401-6.

12. Nozaki K, Shimizu N, Inada K, Tsukamoto T, Inoue M, Kumagai $\mathrm{T}$, et al. Synergistic promoting effects of Helicobacter pylori infection and high-salt diet on gastric carcinogenesis in Mongolian gerbils. Jpn J Cancer Res. 2002;93:1083-9.
13. Ladabaum U. When even people at high risk do not take up colorectal cancer screening. Gut. 2007;56:1648-50.

14. Klein WM, Stefanek ME. Cancer risk elicitation and communication: lessons from the psychology of risk perception. CA Cancer J Clin. 2007;57:147-67.

15. Kinney AY, Hicken B, Simonsen SE, Venne V, Lowstuter K, Balzotti J, et al. Colorectal cancer surveillance behaviors among members of typical and attenuated FAP families. Am J Gastroenterol. 2007;102:153-62.

16. McCaul KD, Branstetter AD, Schroeder DM, Glasgow RE. What is the relationship between breast cancer risk and mammography screening? A meta-analytic review. Health Psychol. 1996;15:423-9.

17. Klabunde CN, Schenck AP, Davis WW. Barriers to colorectal cancer screening among Medicare consumers. Am J Prev Med. 2006;30:313-9.

18. Price PC, Pentecost HC, Voth RD. Perceived event frequency and the optimistic bias: evidence for a two-process model of personal risk judgments. J Exp Soc Psychol. 2002;38:242-52.

19. Duggan A. Understanding interpersonal communication processes across health contexts: advances in the last decade and challenges for the next decade. J Health Commun. 2006; 11:93-108.

20. Ikehara S, Iso H, Date C, Kikuchi S, Watanabe Y, Inaba Y, et al. Salt preference and mortality from stroke and coronary heart disease for Japanese men and women: the JACC study. Prev Med. 2012;54:32-7.

21. Shin JY, Lee DH. Factors associated with the use of gastric cancer screening services in Korea: the Fourth Korea National Health and Nutrition Examination Survey 2008 (KNHANES IV). Asian Pac J Cancer Prev. 2012;13:3773-9.

22. Chang Y, Cho B, Son KY, Shin DW, Shin H, Yang HK, et al. Determinants of gastric cancer screening attendance in Korea: a multi-level analysis. BMC Cancer. 2015;15:336.

23. Kwon YM, Lim HT, Lee K, Cho BL, Park MS, Son KY, et al. Factors associated with use of gastric cancer screening services in Korea. World J Gastroenterol. 2009;15:3653-9.

24. Pate RR, Heath GW, Dowda M, Trost SG. Associations between physical activity and other health behaviors in a representative sample of US adolescents. Am J Public Health. 1996;86:1577-81.

25. Wati YA, Hamied LI, S AM, Sofiatin Y, Roesli RM. Moderate correlation between high salt taste preference and high sodium intake. J Hypertens. 2015;33:e35. 